\title{
Resolução de problemas de elasticidade com carga de domínio pelo Método dos Elementos de Contorno na formulação de dupla reciprocidade
}

\author{
Carlos Andrés Reyna Vera-Tudela
}

Universidade Federal Rural do Rio de Janeiro, Departamento de Matemática, Programa de Pós-Graduação em Modelagem Matemática e Computacional, Rodovia BR 465, Seropédica 23897-000, Rio de Janeiro, Brasil. E-mail: candres@ufrrj.br

Vera-Tudela C.A.R. (2018) Resolução de problemas de elasticidade com carga de domínio pelo Método dos Elementos de Contorno na formulação de dupla reciprocidade. Pesquisa e Ensino em Ciências Exatas e da Natureza, 2(edição especial): 40-51. http://dx.doi.org/10.29215/pecen.v2i2.1039

Editor acadêmico: Saulo Pomponet Oliveira. Recebido: 11 Maio 2018. Aceito: 22 Novembro 2018. Publicado: 26 Novembro 2018.

Resumo: O Método dos Elementos de Contorno transforma equações diferenciais parciais em equações integrais envolvendo somente valores de contorno, após a introdução de funções de ponderação denominadas soluções fundamentais. Quando o problema estudado envolve cargas de domínio deve-se procurar uma solução que preserve a filosofia do método. Para este propósito se utiliza a técnica da Dupla Reciprocidade. Este trabalho ilustra a dupla reciprocidade com a equação de Navier com um termo de peso próprio. Resultados numéricos são apresentados.

Palavras chave: Método dos Elementos de Contorno, Dupla Reciprocidade, Integral de Domínio, Peso Próprio.

Solution of elasticity problems with self-weight loading by the boundary element method under the dual reciprocity formulation

Abstract: The Boundary Element Method transforms partial differential equations that govern the problem domain into integral equations involving only contour values after the introduction of weighting functions called fundamental solutions. When the problem studied involves internal loads, such as self weight, one must search for a solution that preserves the philosophy of the method. For this purpose, the Dual Reciprocity technique is used. This work illustrates dual reciprocity with Navier's equation with a selfweight load term. Numerical results are presented.

Key words: Boundary Element Method, Dual Reciprocity, Domain Integral, Self Weight.

\section{Introdução}

Na Mecânica dos Sólidos é comum estudar as forças de superfície que são definidas como forças que atuam na superfície do corpo que surgem quando os corpos estão em contato e que dão origem a distribuições de tensão (Barber 2010). Forças de superfície também surgem dentro de materiais atuando em superfícies internas. Uma carga de domínio é definida como aquela que age diretamente nas partículas interiores do corpo, e não na fronteira. Como o interior do corpo não é acessível, segue-se necessariamente que as forças do domínio só podem ser produzidas por algum tipo de processo físico que atue "a distância”. Os exemplos mais comuns são forças devidas à gravidade e atração magnética ou eletrostática. Além disso, pode-se formular problemas de elasticidade quase-estática para acelerar corpos em termos de forças corporais, usando o princípio de D’Alembert (Lanczoz 1986). 
A origem da teoria matemática da elasticidade pode-se situar no início do século XIX, principalmente com os trabalhos de Cauchy, o qual introduz os conceitos de tensão e deformação, pouco antes de J. Fourier publicar seu trabalho sobre a propagação do calor. Durante o século XIX aparecem, dentre outros, os trabalhos de Poisson, Lamé, Green, Piola, Kirchhoff e Saint-Venant. Pela metade do século XX, o estudo da elasticidade toma um novo impulso, possivelmente devido às aplicações tecnológicas de novos materiais que foram descobertos. É de especial importância o estudo de placas delgadas na aeronáutica, a propagação de vibrações na construção de maquinário industrial, a propagação de ondas em materiais homogêneos e anisotrópicos em geofísica, a transmissão de calor em astronáutica, entre outros temas (Rodríguez 2012).

O Método dos Elementos de Contorno (MEC) (Brebbia et al. 2012) é relativamente novo se o comparamos com os métodos dos Elementos Finitos (MEF) (Bathe 1996) ou das Diferenças Finitas (MDF) (Burden \& Faires 2008). Elas são técnicas numéricas que têm grande aplicação na solução de problemas governados por equações diferenciais parciais; problemas que na maioria das vezes têm soluções analíticas complicadas ou não conhecidas.

Basicamente pode-se dizer que o Método dos Elementos de Contorno transforma equações diferenciais parciais, que governam o domínio do problema, em equações integrais envolvendo somente valores de contorno, após a introdução de funções de ponderação denominadas soluções fundamentais.

Desta forma, a dimensão do problema é reduzida de uma unidade e somente o contorno do domínio precisa ser discretizado. E esta é uma das grandes vantagens do método porque ao discretizar somente o contorno vamos ter uma menor entrada de dados e como consequência direta um menor gasto computacional, tomando como referência o MEF e o MDF.

O Método da Dupla Reciprocidade (Partridge et al. 1991) é um procedimento apresentado por D. Nardini e C. A. Brebbia em 1982 (Nardini \& Brebbia 1982) para resolver problemas transientes usando soluções fundamentais estáticas, mas que se revelou bastante adequado e eficaz na solução de problemas com ações no domínio. O objetivo da técnica é transformar a integral de domínio, como por exemplo, as forças de volume (Cheng et al. 2001), em uma integral de contorno. Desde que Nardini e Brebbia apresentaram o método, diversos autores vem procurando aplicações em diversos campos das ciências e engenharias: problemas de condução de calor (Behbahani-nia \& Kowsary 2004; Ang 2006), problemas de convecção (Choi 1999), problemas de difusão-convecção (Sarler \& Kuhn 1998; Partridge \& Sensale 2000), problemas de vibração (Davies \& Moslehy 1994; Niku \& Adey 1996), dinâmica da fratura (Albuquerque et al. 2004; Vera-Tudela \& Telles 2005), problema elastoplástico transiente (Owatsiriwong \& Park 2008), entre outros.

A estratégia é de fazer a substituição da grandeza que tem as características de ação de domínio pelo produto de duas outras variáveis, ficando apenas uma delas dependente das grandezas espaciais. A Dupla Reciprocidade surge, então, como uma formulação alternativa do MEC, que mediante o uso de funções auxiliares permite a transformação das integrais de domínio em integrais de contorno, de acordo com os procedimentos clássicos do método.

\section{O Método da Dupla Reciprocidade}

A Equação de Navier, que é uma equação de equilíbrio em termo de deslocamentos, é representada como se vê na equação seguinte:

$$
G u_{j, k k}+\frac{G}{1-2 v} u_{k, k j}=w_{j}
$$

onde $w_{j}$ representa o efeito de domínio que para este trabalho corresponde ao peso próprio, $u$ é o deslocamento, $G$ e $v$ são constantes físicas do material. Na equação (1) e em todas as equações que se seguem no texto é utilizada a notação de Einstein ou notação indicial (Malvern 1969). 
Na equação de Navier são aplicados todos os procedimentos clássicos do MEC (Brebbia et al. 2012), obtendo-se:

$$
\begin{aligned}
& C_{i j}(\xi) u_{j}(\xi)+\int_{\Gamma} p_{i j}^{*}(\xi, x) u_{j}(x) d \Gamma(x)- \\
& \qquad \int_{\Gamma} u_{i j}^{*}(\xi, x) p_{j}(x) d \Gamma(x)=\int_{\Omega} u_{i j}^{*}(\xi, x) w_{j}(x) d \Omega
\end{aligned}
$$

onde $\Gamma$ representa o contorno do problema; $u_{i j}^{*}$ e $p_{i j}^{*}$ na formulação tradicional correspondem à solução fundamental de Kelvin; $C_{i j}$ é um coeficiente relacionado à geometria do corpo e $\xi$ é o ponto fonte.

A solução fundamental para o problema de estado plano num médio elástico infinito é dado pela expressão seguinte (Brebbia et al. 2012),

$$
u_{i j}^{*}(\xi, x)=\frac{-1}{8 \pi(1-v)}\left\{(3-4 v) \ln (r) \delta_{i j}-r,{ }_{i} r, j\right\}
$$

e

$$
p_{i j}^{*}(\xi, x)=\frac{-1}{4 \pi(1-v) r}\left\{\left[(1-2 v) \delta_{i j}+2 r,{ }_{i} r, j\right] \frac{\partial r}{\partial n}-(1-2 v)\left(r,{ }_{i} n_{j}-r,{ }_{j} n_{i}\right)\right\}
$$

onde $r$ é a distância do ponto de aplicação da carga ao ponto em consideração, $n$ representa o vetor normal externo ao corpo e $\frac{\partial \mathrm{r}}{\partial \mathrm{n}}=n_{j} r_{, j}$. A Solução Fundamental é um tipo de função utilizada para resolver equações diferenciais não-homogêneas sujeitas a condições iniciais ou condições de contorno determinadas, e descreve a resposta do sistema físico à função delta de Dirac, que representa um impulso aplicado no ponto $\mathrm{x}$ (magnitude unitária).

O Método da Dupla Reciprocidade (Partridge et al. 1991) é introduzido de forma a expressar a carga de domínio como uma integral de contorno. Para isso, representa-se este termo por um somatório de funções na forma indicial seguinte:

$$
w_{j}(x)=\alpha_{k}^{m} f_{k j}^{m}(x)=\alpha_{k}^{1} f_{k j}^{1}(x)+\alpha_{k}^{2} f_{k j}^{2}(x)+\ldots+\alpha_{k}^{M} f_{k j}^{M}(x),
$$

sendo que, para cada par de índices $k$ e $j, f_{k j}^{m}(x)=f_{k j}\left(\left|x-x^{m}\right|\right)$, onde $x^{1}, \ldots, x^{M}$ são pontos internos, $f_{k j}(r)$ é uma função de aproximação de base radial, e $\alpha_{k}^{1}, \alpha_{k}^{2}, \ldots, \alpha_{k}^{M}$ são coeficientes iniciais desconhecidos.

Desta forma, a integral de domínio passa a ser escrita como:

$$
\int_{\Omega} u_{i j}^{*}(\xi, x) w_{j}(x) d \Omega=\alpha_{k}^{m} \int_{\Omega} f_{k j}^{m}(x) u_{i j}^{*}(\xi, x) d \Omega
$$

Para transformar o lado direito da equação (6) numa integral de contorno, é necessário reescrever $f_{k j}^{m}$ em termos de uma função auxiliar $\Psi_{k j}^{m}$ que precisa satisfazer à equação de Navier com o termo de domínio sendo concentrada no contorno como segue

$$
G \psi_{k j, i i}^{m}+\frac{G}{1-2 v} \psi_{k i, i j}^{m}=\delta_{k j} f^{m}
$$


Substituindo a equação (7) na equação (6), e verificando que, por analogia as mesmas operações já desenvolvidas na formulação tradicional podem ser empregadas, obtem-se:

$$
C_{i j}^{l} u_{j}^{l}+\int_{\Gamma} p_{i j}^{*} u_{j} d \Gamma-\int_{\Gamma} u_{i j}^{*} p_{j} d \Gamma=\left(C_{i j}^{l} \psi_{k j}^{l m}+\int_{\Gamma} p_{i j}^{*} \psi_{k j}^{m} d \Gamma-\int_{\Gamma} u_{i j}^{*} \eta_{k j}^{m} d \Gamma\right) \alpha_{k}^{m}
$$

Nesta expressão o termo $\eta_{k j}^{m}$ corresponde ás forças de superfície recíprocas, referentes à função auxiliar $\Psi_{k j}^{m}$, correspondente aos deslocamentos.

As funções auxiliares de interpolação $\Psi^{m}{ }_{k j}$ são dadas por $\Psi_{k j}^{m}(x)=\Psi_{k j}\left(\left|x-x^{m}\right|\right)$, sendo $\Psi_{k j}(r)$ definida pela seguinte expressão (Vera-Tudela 2003):

$$
\psi_{k j}(r)=\frac{1}{30(1-v) \mu}\left[\left(3-\frac{10 v}{3}\right) \delta_{k j}-r, k r, j\right] r^{3},
$$

enquanto que $\eta_{k j}^{m}(x)=\eta_{k j}\left(\left|x-x^{m}\right|\right)$, onde

$$
\eta_{k j}(r)=\frac{1}{15(1-v)}\left[(4-5 v) r, k n_{j}-(1-5 v) r,{ }_{j} n_{k}+\left[(4-5 v) \delta_{k j}-r,{ }_{j} r, k\right] \frac{\partial r}{\partial n}\right] r^{2} .
$$

\section{Implementação Numérica}

Uma vez obtida a equação integral de contorno é preciso discretizá-la para então resolvêla (Vera-Tudela 2003). Para a discretização da equação (8), divide-se o contorno em $J$ elementos $\Gamma_{j}$. A esses elementos estão associados valores nodais de deslocamentos e forças de superfície, em função dos quais a variação destas grandezas dentro do elemento depende das funções de interpolação $N$ utilizadas e que, por sua vez, dependem do número de nós funcionais $n$ dos elementos. Desta forma, pode-se expressar os valores de deslocamentos $u_{j}$ da equação (8) na forma matricial seguinte:

$$
u_{j}=\left\{\begin{array}{l}
u_{1} \\
u_{2}
\end{array}\right\}=N u^{(n)}
$$

e para forças de superfície $p_{j}$ :

$$
p_{j}=\left\{\begin{array}{l}
p_{1} \\
p_{2}
\end{array}\right\}=N p^{(n)}
$$

onde

$$
N=\left[\begin{array}{cccccc}
\phi_{1} & 0 & \phi_{2} & 0 & \phi_{3} & 0 \\
0 & \phi_{1} & 0 & \phi_{2} & 0 & \phi_{3}
\end{array}\right]
$$

Também, para elemento quadrático, tem-se: 


$$
u^{(n)}=\left\{\begin{array}{l}
u_{1}^{1} \\
u_{1}^{2} \\
u_{2}^{1} \\
u_{2}^{2} \\
u_{3}^{1} \\
u_{3}^{3}
\end{array}\right\}, p^{(n)}=\left\{\begin{array}{l}
p_{1}^{1} \\
p_{1}^{2} \\
p_{2}^{1} \\
p_{2}^{2} \\
p_{3}^{1} \\
p_{3}^{3}
\end{array}\right\}
$$

Observe-se que os procedimentos aplicados sobre $u$ e $p$, também serão realizados sobre $\Psi$ e $\eta$. Substituindo as equações (11) e (12) na equação (8) tem-se,

$$
\begin{gathered}
C u+\sum_{j=1}^{J}\left(\int_{\Gamma_{j}} p^{*} N d \Gamma\right) u^{(n)}-\sum_{j=1}^{J}\left(\int_{\Gamma_{j}} u^{*} N d \Gamma\right) p^{(n)}= \\
\sum_{j=1}^{J}\left(C \psi^{(n)}+\sum_{j=1}^{J}\left(\int_{\Gamma_{j}} \eta^{*} N d \Gamma\right) \psi^{(n)}-\sum_{j=1}^{J}\left(\int_{\Gamma_{j}} \psi^{*} N d \Gamma\right) \eta^{(n)}\right) \alpha^{n} .
\end{gathered}
$$

O elemento de contorno $\Gamma_{j}$ varia de acordo com a função de interpolação adotada, neste trabalho é considerada a função de interpolação linear com dois nós geométricos por elemento.

Durante a montagem do sistema indicado pela equação (15), cada uma das integrais será calculada numericamente sempre que o ponto fonte $\xi$ não pertencer ao elemento $\Gamma_{j}$ sobre o qual está sendo efetuada a integração. Este cálculo se dará através da integração numérica de Gauss dada por,

$$
\int_{-1}^{1} f(\eta) d \eta=\sum_{k=1}^{N P I} f\left(\eta_{k}\right) w_{k}
$$

onde $\eta_{k}$ é a coordenada adimensional do k-ésimo ponto de integração, $w_{k}$ é o fator de peso associado ao ponto $k$ e NPI é o número total de pontos de integração utilizado. Desta forma, trabalha-se com as parcelas da equação (15) como segue,

$$
\int_{\Gamma_{j}} p^{*} N d \Gamma=\int_{-1}^{1} p^{*} N|J| d \eta \cong \sum_{k=1}^{N P I}|J|_{k} w_{k} N_{k} p_{k}^{*},
$$

e

$$
\int_{\Gamma_{j}} u^{*} N d \Gamma=\int_{-1}^{1} u^{*} N|J| d \eta \cong \sum_{k=1}^{N P I}|J|_{k} w_{k} N_{k} u_{k}^{*}
$$

A equação integral discretizada é aplicada repetidamente considerando o ponto fonte $\xi$ situado coincidentemente com todos os pontos nodais existentes. Um sistema de $2 n$ equações algébricas é gerado e envolve $2 n$ valores nodais de deslocamento e força de superfície. Ainda é necessário levar este sistema para uma forma matricial e para isso coloca-se da forma a seguir,

$$
\sum_{j=1}^{J}\left(\int_{\Gamma_{j}} p^{*} N d \Gamma\right) u^{(n)}=\sum_{j=1}^{J} h_{j} u^{(n)}
$$


Similarmente

$$
\sum_{j=1}^{J}\left(\int_{\Gamma_{j}} u^{*} N d \Gamma\right) p^{(n)}=\sum_{j=1}^{J} g_{j} p^{(n)}
$$

O sistema fica reduzido na forma a seguir,

$$
C u^{(n)}+\sum_{j=1}^{J} h_{j} u^{(n)}-\sum_{j=1}^{J} g_{j} p^{(n)}=\left(C \psi^{(n)}+\sum_{j=1}^{J} h_{j} \psi^{(n)}-\sum_{j=1}^{J} g_{j} \eta^{(n)}\right) f^{-1} w .
$$

Resulta, então, um sistema de equações matriciais na forma

$$
\mathbf{H u}-\mathbf{G p}=(\mathbf{H} \psi-\mathbf{G} \eta) \mathbf{f}^{-\mathbf{1}} \mathbf{w} .
$$

\section{Resultados e Discussão}

O software foi desenvolvido utilizando linguagem de programação Fortran 90, utilizando o compilador Intel Visual Fortran 13.0. O computador utilizado foi um notebook Dell Inspiron 5537, com processador Intel Core i7, 16GB de memória RAM e sistema operacional Windows 10.

\section{Exemplo 1}

Neste exemplo tem-se a geometria mostrada na Figura 1. A viga está sob o efeito de carregamento dado pelo peso próprio. A viga tem comprimento de 24 e altura de 6, o módulo de Young é igual a 10000 e o coeficiente de Poisson é igual a 0. Os resultados são dados para um ponto $\mathrm{P}$ no centro do extremo livre. Os testes para a formulação de elementos de Contorno são realizados utilizando malhas com 12, 24 e 48 elementos quadráticos.

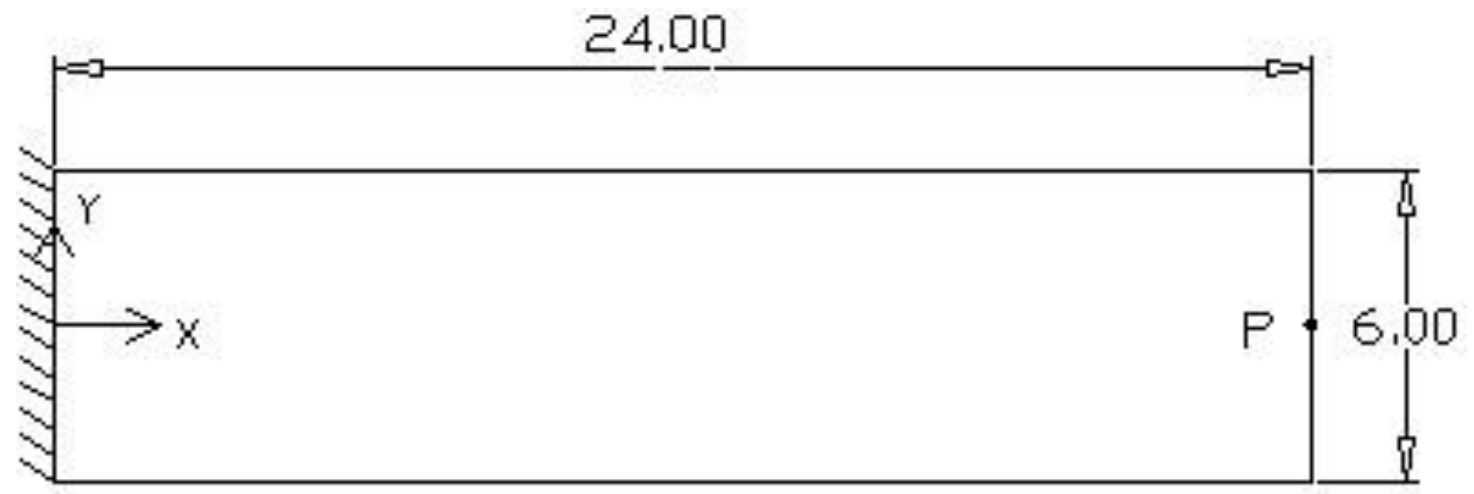

Figura 1. Viga Engastada com carga de domínio peso próprio.

Como solução de referência, usa-se a obtida pelo Método dos Elementos Finitos com ajuda do Software ANSYS 5.4. Para o ponto P o deslocamento é -1.4606u. A malha tem 576 elementos quadrangulares quadráticos.

Os resultados obtidos estão mostrados na Tabela 1 e pode-se observar que existe aproximação de resultados com erro final de $2.11 \%$ da solução de referência.

Tabela 1. Deslocamento do ponto $\mathrm{P}$ da viga.

\begin{tabular}{cc}
\hline Elementos & BEM \\
\hline 12 & $-1.3993 \mathrm{u}$ \\
24 & $-1.4208 \mathrm{u}$ \\
48 & $-1.4296 \mathrm{u}$ \\
\hline
\end{tabular}


Trabalha-se agora com a malha de 24 elementos, considerando $M=5,17,23$ e 35 pontos internos distribuídos de forma uniforme em todo o domínio. Observa-se que a inclusão dos pontos internos melhora muito a tendência dos resultados, com um erro final de $1.12 \%$. Na Tabela 2 estão indicados os valores de deslocamento do ponto $\mathrm{P}$ com o incremento dos pontos internos.

Tabela 2. Variação do deslocamento do ponto P com o incremento dos pontos internos.

\begin{tabular}{ccc}
\hline PI & Deslocamento & Erro (\%) \\
\hline 0 & -1.4208 & 2,72 \\
5 & -1.4361 & 1.68 \\
17 & -1.4416 & 1.30 \\
23 & -1.4435 & 1.17 \\
35 & -1.4443 & 1.12 \\
\hline
\end{tabular}

\section{Exemplo 2}

Este exemplo consiste na análise de uma barra, com seção transversal constante, fixa no extremo superior e submetida ao efeito da carga de domínio que, neste caso, é o peso próprio.

A Figura 2 mostra as características geométricas do sistema.

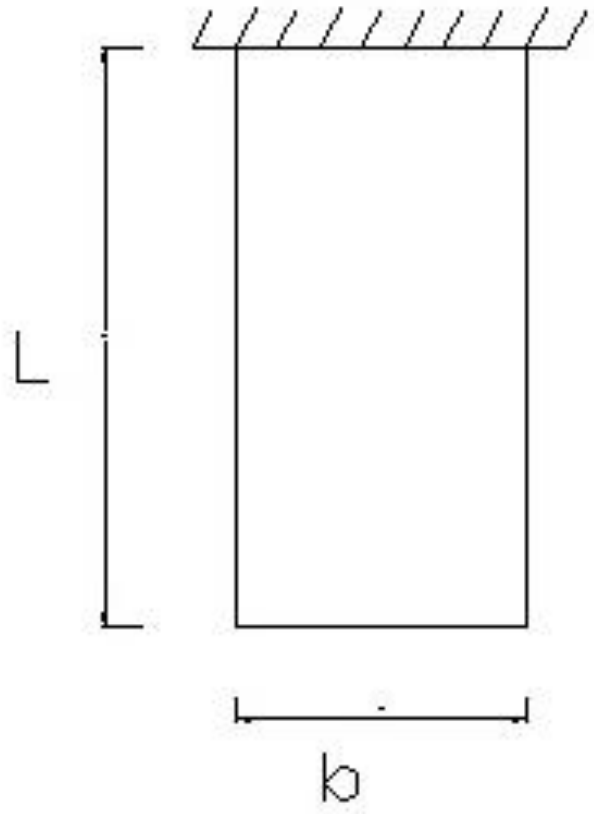

Figura 2. Barra de seção reta com carga de domínio variável linearmente.

A expressão teórica para o cálculo de deslocamentos em uma barra sob ação do peso próprio é dada da seguinte maneira (Vera-Tudela 2003):

$$
u=\frac{\rho g}{E}\left(\frac{x^{2}}{2}-L x\right)
$$

Nas simulações doravante apresentadas é admitido que $\rho g=1, E / \rho=10^{4}$ e $v=0$.

Neste exemplo, foram usadas as funções de interpolação lineares. Na Tabela 3 coloca-se um resumo dos dados geométricos e do material. Neste exemplo, ressalta-se que o interesse é comparar os deslocamentos máximos (na direção Y) da barra modificando só o seu comprimento. 
Em todos os casos são utilizados os nós duplos nos cantos da barra e no engaste são prescritos deslocamentos nulos nas duas direções X e Y.

Tabela 3. Dados geométricos da barra de seção constante.

\begin{tabular}{cccc}
\hline L/b & L & b & Número de Nós \\
\hline $\mathbf{1}$ & 9 & 9 & $24,34,64,84$ \\
$\mathbf{2}$ & 18 & 9 & $24,34,64,84$ \\
$\mathbf{4}$ & 36 & 9 & $24,34,64,84$ \\
\hline
\end{tabular}

Na Tabela 4 observa-se os valores de erro no cálculo de deslocamento na extremidade livre em função do refinamento da malha para a relação $\mathrm{L} / \mathrm{b}=1$. Cabe mencionar que é preciso apresentar tanto os valores do ponto de máximo deslocamento quanto a média dos valores de deslocamento na extremidade livre, isto porque a solução analítica tomada como referência é uma solução teórica aproximada, onde uma série de fenômenos físicos pertinentes a um problema elástico real não são considerados, como: efeito dos cantos, efeito do engastamento e coeficiente de Poisson.

Tabela 4. Erro máximo e médio para $\mathrm{L} / \mathrm{b}=1$.

\begin{tabular}{ccc}
\hline Núm. Nós & Erro u máximo & Erro u médio \\
\hline 24 & 3.7531 & 7.2840 \\
36 & 5.9259 & 6.1432 \\
64 & 10.4444 & 5.4815 \\
84 & 11.4074 & 5.4070 \\
\hline
\end{tabular}

Na Tabela 5 pode-se observar que o aumento no comprimento do problema físico melhora os resultados, $\mathrm{L} / \mathrm{b}=2$, como se pode conferir observando os valores dos erros. Isso é função da melhoria da representatividade do modelo matemático com relação ao problema físico, onde as condições de engastamento passam a perturbar menos a resposta.

Ainda neste ponto não está se analisando como a inclusão de pontos internos poderia influenciar os resultados; este é um estudo posterior para complementar essa análise.

Tabela 5. Erro máximo e médio para $\mathrm{L} / \mathrm{b}=2$.

\begin{tabular}{ccc}
\hline Núm. Nós & Erro u máximo & Erro u médio \\
\hline 24 & 6.5617 & 6.5895 \\
36 & 3.8086 & 5.0877 \\
64 & 2.1111 & 4.3451 \\
84 & 2.0370 & 4.2580 \\
\hline
\end{tabular}

Na Tabela 6 analisa-se o último exemplo comparativo dessa série para a relação $L / b=4$, que corresponderia a uma barra mais comprida.

Praticamente o erro convergiu para um nível bastante aceitável e os resultados obtidos demostram que a teoria tradicional de barras apresentado pela Resistência dos Materiais considera que o comprimento é bem maior que a largura (Popov 1999). Os resultados mostram que o método fica estável e apresenta resultados também coerentes.

Tabela 6. Erro máximo e médio para $\mathrm{L} / \mathrm{b}=4$.

\begin{tabular}{ccc}
\hline Núm. Nós & Erro u máximo & Erro u médio \\
\hline 24 & 1.8544 & 2.0431 \\
36 & 1.6499 & 1.3195 \\
64 & 0.0422 & 0.3442 \\
84 & 0.0853 & 0.2624 \\
\hline
\end{tabular}




\section{Exemplo 3}

Neste exemplo estuda-se a barra de seção constante, mas neste caso, a carga de domínio é variável desde um valor máximo no extremo superior (engaste) até o valor nulo no extremo inferior (Figura 3).

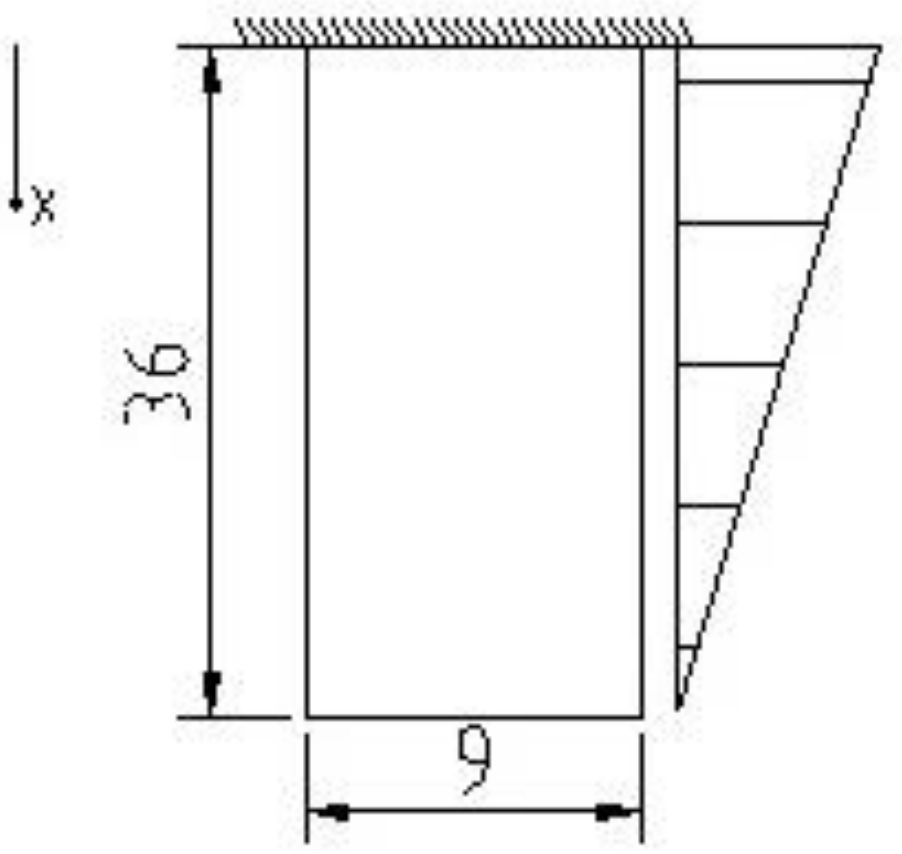

Figura 3. Barra de seção reta com carga de domínio variável linearmente.

A geometria da barra corresponde à relação $\frac{L}{b}=4(L=36, b=9)$; as malhas consideradas têm 36,64 e 84 nós, sem pontos internos.

A expressão que representa os deslocamentos pode ser obtida da teoria da Resistência dos Materiais pela seguinte fórmula:

$$
u=-\frac{\rho g}{E L} \frac{x^{3}}{6}
$$

Considera-se para fins de simulação numérica que $\rho g=1$.

Na Tabela 7, pode-se observar que os valores dos erros absolutos e percentual diminuem conforme aumenta o número de nós de contorno. Isto ocorre por ser a carga de domínio variável, e assim o campo de deslocamentos é cúbico. Para analisar a convergência dos resultados pode-se observar na Figura 4 um gráfico mostrando no eixo das abcissas o número de nós e no eixo das ordenadas o erro percentual. Neste gráfico foi adicionada uma linha de tendência. Pode-se observar que para 202 nós de contorno o valor do erro percentual diminui para $0.23 \%$. Com estes valores de erro, é interessante estudar, em trabalhos futuros, o efeito de considerar pontos internos na barra reta, pois a introdução destes pontos melhoram os resultados (Partridge et al. 1991).

Tabela 7. Erros em porcentagem para a barra reta com carga variável.

\begin{tabular}{ccc}
\hline Núm. Nós & Erro absoluto & Erro percentual \\
\hline 36 & 0.0013 & 6.45 \\
64 & 0.0011 & 5.09 \\
84 & 0.0010 & 4.74 \\
\hline
\end{tabular}


Neste exemplo pode-se observar a mesma tendência do exemplo anterior onde o refinamento da malha melhora os resultados, comparados com o valor teórico. Assim, os resultados estão confirmando, mais uma vez, a convergência dos resultados com o aumento do refinamento da malha.

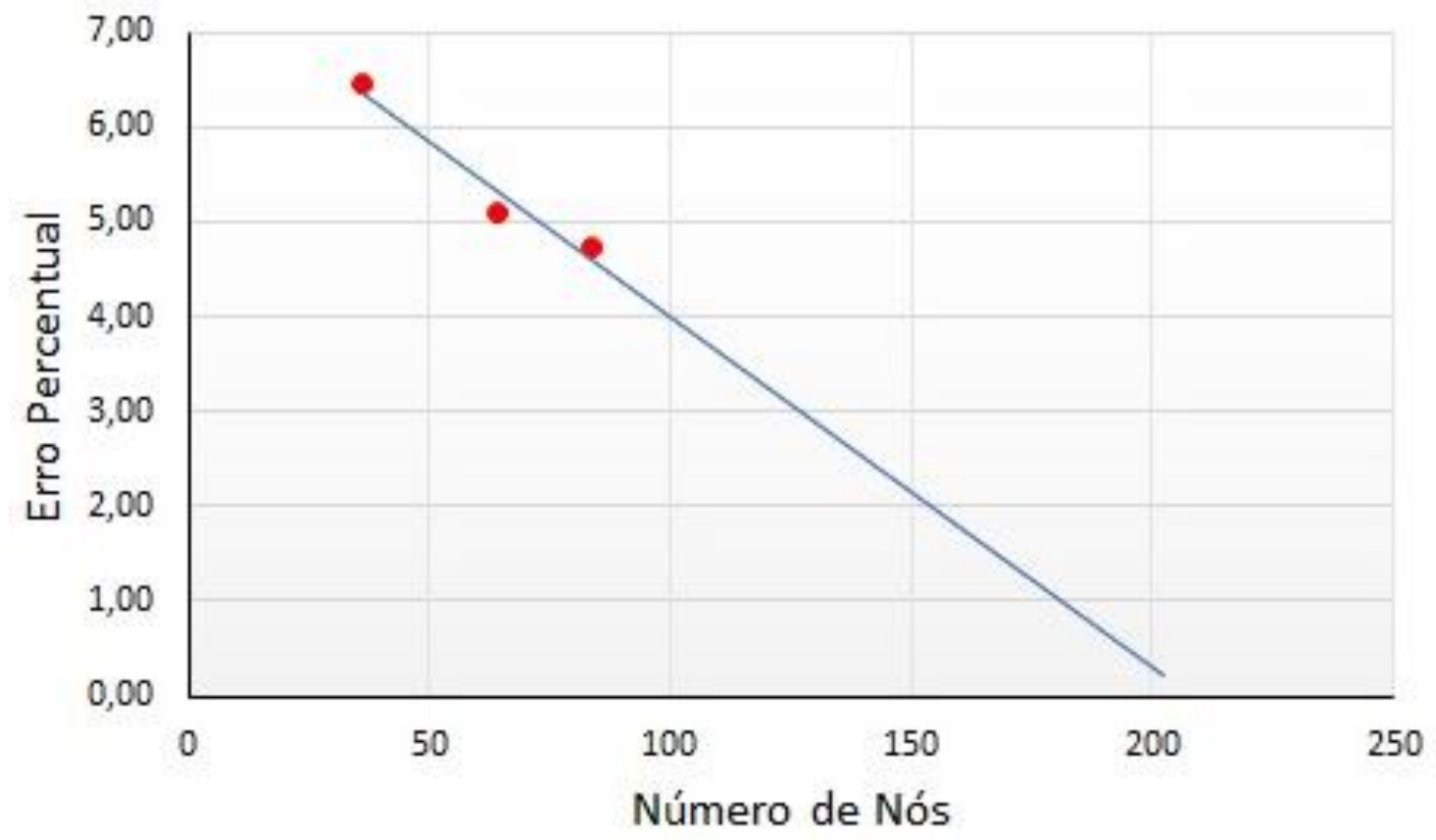

Figura 4. Gráfico de Regressão linear Número de Nós e Erro Percentual.

\section{Conclusões}

Foi apresentado o Método dos Elementos de Contorno com a Técnica da Dupla Reciprocidade para resolver problemas com carga de domínio, para o caso específico do peso próprio.

Também é estudada a importância da inclusão de pontos internos com o objetivo de melhorar a aproximação dos resultados. Para o exemplo 1, os resultados sem pontos internos apresentam um erro de pouco mais de $2 \%$ enquanto que a inclusão de pontos internos melhora os resultados com redução do erro para pouco mais de $1 \%$.

Nos exemplos 2 e 3 são conhecidos os resultados analíticos para deslocamento. Assim, são comparados somente os valores dos erros para mostrar, principalmente, como o refinamento da discretização melhoram os valores obtidos. Como apresentado no exemplo 1, a inclusão de pontos internos ajuda a melhorar os resultados.

É importante destacar que a Técnica da Dupla Reciprocidade permite resolver o problema numericamente seguindo a filosofia do Método dos Elementos de Contorno que é a utilização de integrais definidas unicamente no contorno, embora as cargas devidas a peso próprio formem integrais de domínio.

Para trabalhos futuros sugere-se a redução progressiva do tamanho dos elementos de contorno, o que traz como consequência o aumento no número de elementos e nós de contorno. Neste caso a exigência computacional aumenta consideravelmente. $\mathrm{O}$ aumento progressivo do número de pontos internos, também, deve ser avaliado e relacionado com o nível de erro do método e/ou formulação apresentada.

\section{Agradecimentos}

Aos avaliadores anônimos pela revisão crítica do manuscrito. 


\section{Referências}

Albuquerque E.L., Sollero P. \& Aliabadi M.H. (2004) Dual boundary element method for anisotropic dynamic fracture mechanics. International Journal for Numerical Methods in Engineering, 59(9): 1187-1205. Doi: 10.1002/nme.912

Ang W.T. (2006) A dual-reciprocity boundary element approach for solving axisymmetric heat equation subject to specification of energy. Engineering Analysis with Boundary Elements, 32: 210-215.

Barber J.R. (2010) Body Forces (p. 91-108). In: Barber J.R. (Ed.). Elasticity. Solid Mechanics and Its Applications. Volume 172. Springer: Dordrecht. 534 p.

Bathe K.J. (1996) Finite Element Procedures. New Jersey: Prentice Hall. 1037 p.

Behbahani-nia A. \& Kowsary F. (2004) A dual reciprocity BE-based sequential function specification solution method for inverse heat conduction problems. International Journal of Heat and Mass Transfer, 47: 1247-1255. Doi: 10.1016/j.ijheatmasstransfer.2003.09.023

Brebbia C.A., Telles J.C.F. \& Wrobel L.C. (2012) Boundary Element Techniques. Berlin: Springer. $464 \mathrm{p}$.

Burden R.L. \& Faires J.D. (2008) Análise Numérica. São Paulo: Cengage do Brasil. 736 p.

Cheng A.H.D., Cheng C.S., Goldberg M.A. \& Rashed Y.F. (2001) BEM for thermoelasticity and elasticity with body force - A revisit. Engineering Analysis with Boundary Elements, 25: 377387. Doi: 10.1016/S0955-7997(01)00032-7

Choi C. Y. (1999) Dual reciprocity boundary element analysis for the laminar forced heat convection problem in concentric annulus. KSME International Journal, 13: 496-503. Doi: 10.1007/BF02947719

Davies T.W. \& Moslehy F.A. (1994) Modal analysis of plates using the dual boundary element method. Engineering Analysis with Boundary Elements, 14: 357-362. Doi: 10.1016/09557997(94)90066-3

Lanczoz C. (1986) The Variational Principles of Mechanics. Toronto: Dover Publications. 464 p.

Malvern L.E. (1969) Introduction to the Mechanics of a Continuous Medium. New Jersey: Prentice-Hall. $771 \mathrm{p}$.

Nardini D. \& Brebbia C.A. (1982) A new approach to free vibration analysis using boundary elements. Applied Mathematical Modelling, 7: 157-162. Doi: 10.1016/0307-904X(83)90003-3

Niku S.M. \& Adey R.A. (1996) Computational aspect of the dual reciprocity method for dynamics. Engineering Analysis with Boundary Elements, 18: 43-61.

Owatsiriwong A. \& Park K.H. (2008) A BEM formulation for transient dynamic elastoplastic analysis via particular integrals. International Journal of Solids and Structures, 45: 25612582. Doi: 10.1016/j.jijsolstr.2007.12.009

Partridge P.W. \& Sensale B. (2000) The method of fundamental solution with dual reciprocity for diffusion and diffusion-convection using subdomains. Engineering Analysis with Boundary Elements, 24: 633-641. Doi: 10.1016/S0955-7997(00)00043-6

Partridge P.W., Brebbia C.A. \& Wrobel L.C. (1991) The Dual Reciprocity Boundary Element Method. Berlin: Computational Mechanics Publications. 276 p.

Popov E.P. (1999) Mechanics of Solids. New Jersey: Prentice Hall. 888 p.

Rodríguez C.P. (2012) Método de elementos de contorno de reciprocidad dual aplicado a la termoelasticidad anisótropa desacoplada. Tese de Doutorado, Departamento de Ingeniería Mecánica y de los Materiales, Escuela Técnica Superior de Ingeniería, Universidad de Sevilla, Sevilla.

Sarler B. \& Kuhn G. (1998) Dual reciprocity boundary element method for convective-diffusive solid-liquid phase change problems. Part I - Formulation. Engineering Analysis with Boundary Elements, 21: 53-63. Doi: 10.1016/S0955-7997(97)00112-4

Vera-Tudela C.A.R. (2003) Formulações Alternativas do MEC para Problemas Elastodinâmicos de Mecânica da Fratura com o uso da Função de Green Numérica. Tese de Doutorado, COPPE - Universidade Federal do Rio de Janeiro, Rio de Janeiro. 
Vera-Tudela C.A.R. \& Telles J.C.F. (2005) A numerical Green's function and dual reciprocity BEM method to solve elastodynamic crack problems. Engineering Analysis with Boundary Elements, 29: 204-209. Doi: 10.1016/j.enganabound.2005.01.004 